\title{
Research on Network Undergraduates Sports Service System Based on Multi-Agent Technology
}

\author{
Cong Ding \\ Department of Physical education, North-west University, Xi'an, 710069, China \\ dddingcong@163.com
}

Keywords: College campuses, Undergraduates, Sports service, Multi-agent

\begin{abstract}
To reinforce sports informatization management and the sports service quality on college campuses, this paper researches combined multi-agent technology, and structures undergraduate sports service system framework. It has elaborated functions of every feature and workflow of the system, and puts forward agent design procedure based on JADE. What is more, it also adopts FIPAACl linguistic norms between Agents communication and proposes some advices which based on the fundamental of Agent.
\end{abstract}

\section{Introduction}

Many studies have shown that undergraduates physical quality is worrying, the body quality development was not balance, body function became down, levels of constitution health was lower national levels overall [1,2]. It is important that the physical exercise model and scientific guiding on exercise for college students should be established quickly in order to provide the benefit method for improving their sports. In recent years, researches on School Physical Education under the information environment have become quite popular [3]. With the rapid development of mobile Internet, the application of artificial intelligence technology and data technology as the core of the network technologies, helps more advanced technology play a breakthrough role in the field of school sports [4]. To build a system network sports service system for different undergraduates' different demand, offering individual service, changing passive service to active service and assisting client screen out accuracy information are very necessary. Individual sports service system can offer appropriate information in proper timing according to undergraduates' preference and their browsing custom. This can improve information platform information utilization and undergraduates' satisfaction greatly.

Agent technology derives from distributed artificial intelligence(DAI) field. The technology has developed more and more important function [5] in the nowadays computer main current field based network distributed computing. This paper plans to adopt multi-Agent technology from information service view to design undergraduates sports service system. It promotes information construction of college campuses in abundant multimedia means to improve sports service quality.

\section{General design of undergraduates sports service system}

Adopting Agent technology and through constantly independent study, we should find undergraduate interests and make matching of multimedia document information to meet students individuation demand of sports searching. The general framework is like Fig. 1.

The system mainly includes client interface Agent, filter sort Agent, interest study Agent, monitoring Agent and information searching Agent. In the five modules, interest study Agent makes interaction with client, information searching Agent and filter sort Agent can make proceed search return result except of communication and cooperation internally. 


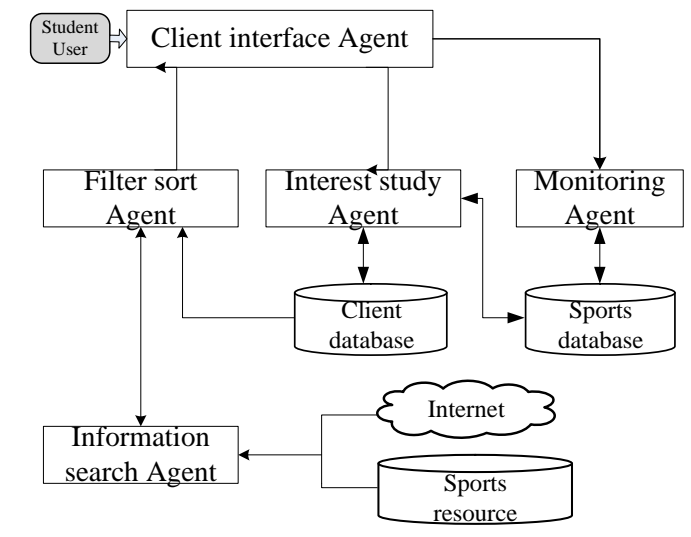

Fig. 1 The framework of undergraduates sports service system

\section{System mainly function module}

\subsection{Client interface Agent.}

The agent offers client some functions like registration, loading, checking system pushed information, submitting data request and undergraduate feedback. It is a platform for undergraduates making communication with the system. Client interface Agent would be activated after the client logs in the system. And it is responsible for transmitting filtered information to the client. In the meanwhile, it also can collect undergraduates' feedback opinions and then push them to the interest study Agent, which can update the client interest model.

There are two ways of undergraduate feedback. The one is that dominant undergraduate comments on the pushing information on the client-side interface. The other one is to adjust the interest according to the undergraduate browsing behaviors. Undergraduate browsing and operating the related information is a recessivity feedback. Analyzing the feedback, we can make the system pushed content to be more pertinence, and conforming to undergraduates' individuation request.

\subsection{Filter sort Agent.}

The agent is an intermediary agent between information source and client. When information source and client both do not grasp adverse request knowledge, we can make filter of the search engine result through interest sorting algorithm and client interest model, which is the key for realizing the active service function of intellectual. The traditional search engine only queries by searching keywords. This certainly would bring up a mass of redundant information. Thus, how to extract the client interest from the information is the key issue that the system tries to solve. Hence, we need to make filter and sorting the searching result combined with undergraduate interest database, and then return to the client. The route chart of the filter sort Agent is like the Fig. 2.

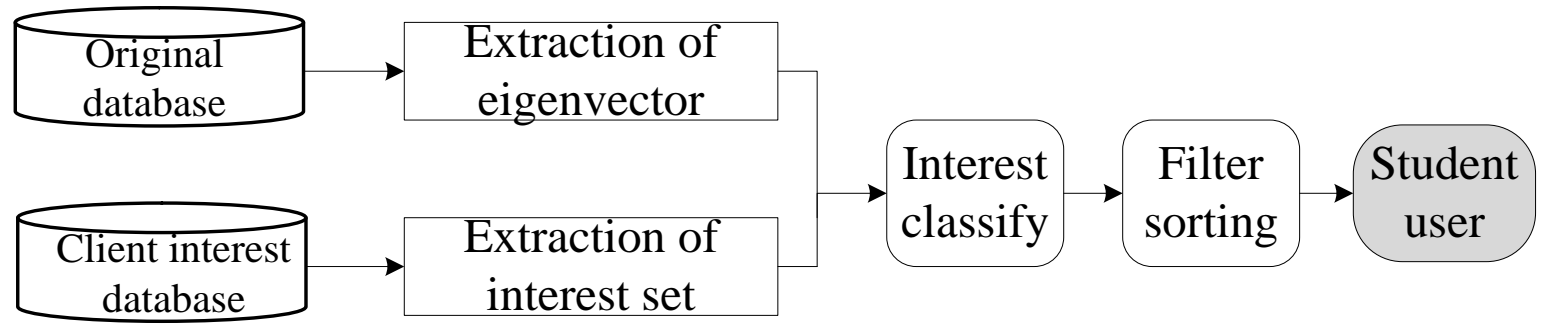

Fig. 2 The route chart of filter sort Agent

The filter sort Agent has made pretreatment for the information search Agent result, and then extracted the document eigenvector. Then it extracted the client interest set from the client interest database. At last, it made filter and sorting according to the interest classify result to push the result, which should be satisfied with a certain threshold value, to the client. 


\subsection{Interest study Agent.}

The agent is a foundation to realize personalized information active service. It is responsible for making communication with client interface Agent to obtain useful information which can reflect client interests, and then adjusts client interest model. Interest study Agent can obtain client interest information from two aspects. The one is the feedback comment that the client making for the watched content and the client registration. The other one is an implicit expression information. It can obtain some useful information by recording undergraduates' behaviors like collecting, browsing, downloading and deleting the related themes. There are two phases for the client interest model establishment. The one is according to undergraduate offering the original registration information to establish the interest eigenvector. And then it uses the learning function of interest study Agent to analyze the feedback information and behavioral preference. This can modify the client interest model and make weight adjustment for the different feature words of client eigenvector. The weight of interest keyword is higher; client is more interested in the information that the keyword represents. Thus, the system can feedback client personalized request to some extent.

\subsection{Monitoring Agent.}

The agent is used to record client behavior act. When the client log in the system, the monitoring Agent has been activated, and recorded the client all activities like information collecting, browsing and downloading. The system would express the behavior information in a quantitative way, and store it into the locality database to adjust client interest model. Monitoring Agent also makes dynamic monitoring for the system offered personal interest station. If it finds the station was changed, it would inform the information search Agent to fetch information, and then let filter sort Agent manage it to push to the client.

\subsection{Information search Agent.}

The agent can accept other Agent modules' searching request. And then it would make pretreatment analysis about the request information. Then it submits the information to the search engine which can submit the result document to the filter sort Agent for the re-treatment. Information search Agent has many jobs. It can make pretreatment for the information searching keyword, and adopt word segmentation technology to do word segmentation of the searching request for extracting the keyword. To ensure normal use of the searching function, it should make communication with the system formulated searching engine. The general searching engine is composed by searcher, indexer and interface and other parts. Information search Agent need to overcome the different between search engine interface, searching technology and result represent to obtain multiple searching engine results. The specific function of the information search Agent is showed on Fig. 3.

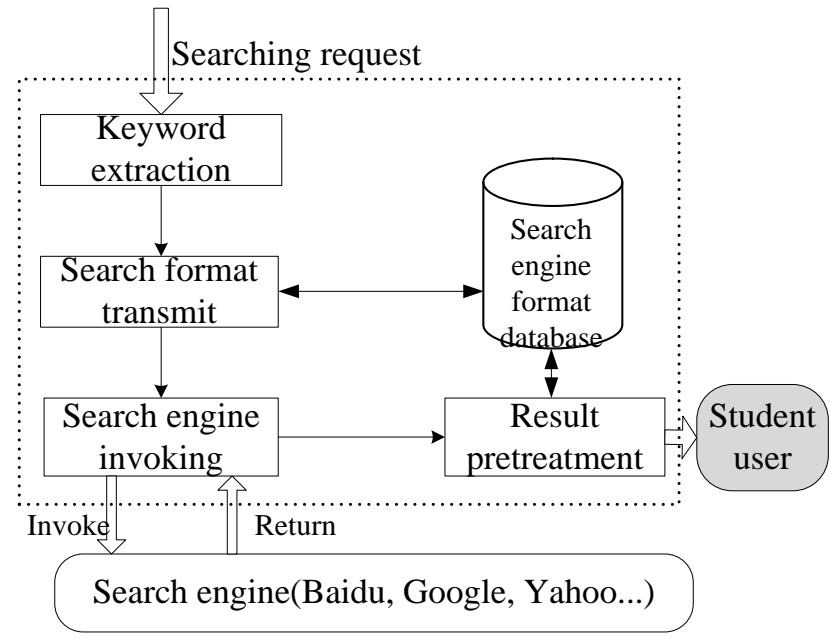

Fig. 3 The specific function of information search Agent 
Keyword extraction model is to use word segmentation technology to process the searching request, and then extract the searching keyword. Search format transmit model is combined with different engine searching format specific requirement of engine format database to transmit the keyword to the format which search engine can handle.

Search engine invoking model can coordinate system default or client appointed search engine to adopt multithreading concurrent execution to let them process searching request at the same time and extract the return result.

Result pretreatment model need to unitize process the result because of different search engine result format. In addition, the model can delete the repeated information and invalid interlinkage of the results.

\section{Implementation suggestion}

The system can adopt the Agent design based on JADE (Java Agent Development Framework). JADE is an intelligent development platform to exploit standard software frame based on Agent, conformed to FIPA intelligent multi-agent system. JADE can manage the affair which is independent of specific application like information passing, coding and analyzing, and Agent life cycle management. Each Agent of the system makes communication by FIPAACL language specification, and based on the foundation of information.

\section{Conclusion}

The research designed the overall architecture of undergraduates sports service system. And it put forward implementation scheme based on multi-agent technology. It made structural division for the mainly Agent function models. And it elaborated specific function and working process of every function model, which included client interface Agent, filter sort Agent, interest study Agent, monitoring Agent and information search Agent five models. They provide some basis and guidance for the follow-up system implementation.

\section{References}

[1] Yang Peiji, Zhou Xuebing, Yu Xiao-dong. A Research on the current situation of university stuendts activity after school in parts of china[J]. Journal of Beijing sport university. 2009, 32 (7):91-93.

[2] Ping Liang. College sports and health curriculum[M]. China Electric Power Press, first ed. Beijing, 2009.

[3] Wu Ruoxi, Wang Qingjun, Fitness APP: development status, problems and countermeasures [J]. Journal of Shandong Sport University. 2015,31(6):18-22.

[4] Changliang Du, Shaodan Li. Sports training theory based on complexity sience [J]. Journal of Beijing Sport University, 2009,32(7):146-149.

[5] Mouritsen, J., Larsen, H. The 2nd wave of knowledge management: The management control of knowledge resources through intellectual capital information[J]. Management Ac-counting Research, 2005,16(3):371-394. 